\title{
Advanced Sarcoma
}

National Cancer Institute

\section{Source}

National Cancer Institute. Advanced Sarcoma. NCI Thesaurus. Code C153185.

A sarcoma that has spread extensively to other anatomic sites or is no longer responding to treatment. 